\title{
Efecto de la Terapia Láser de Baja Potencia sobre el Hueso Alveolar Dañado
}

\author{
The Low Level Laser Therapy Effect on Alveolar Bone Damaged \\ "María José Landaeta Bendezú; *Iván Claudio Suazo Galdames; *Mario Cantín López; \\ "Ignacio Javier Roa Henriquez \& *"Daniela Alejandra Zavando Matamala
}

LANDAETA, B. M. J.; SUAZO, G. I. C.; CANTIN, L. M.; ROA, H. I. J.; ZAVANDO, M. D. A. Efecto de la terapia láser de baja potencia sobre el hueso alveolar dañado. Int. J. Morphol., 26(3):639-642, 2008.

RESUMEN: La terapia con Láser de baja potencia ha demostrado tener propiedades analgésicas antiinflamatorias, bioestimulantes y promotoras de la respuesta tisular al daño. El propósito de este estudio fue determinar el efecto que el láser de baja potencia tiene sobre el hueso alveolar dañado. Se utilizaros 13 ratas Sprage Dawley, en las cuales se realizó una lesión estandarizada del hueso alveolar, posterior a lo cual una muestra aleatoria de 7 ratas fue sometida a un protocolo de irradiación de $6 \mathrm{~J} / \mathrm{cm} 2$, tres veces por semana durante cuatro semanas. Las muestras obtenidas del sitio lesionado en ratas expuestas y no expuestas a la terapia fueron procesadas para hematoxilina eosina, contabilizándose el número de osteonas al microscopio óptico con aumento de 40x. Los resultados muestran un aumento en el número de osteonas en el grupo irradiado, diferencia que resultó estadísticamente significativa $(\mathrm{p}<0,01)$, con una alta fuerza de asociación estadística $(\mathrm{O} . \mathrm{R}=5,6)$. Estos resultados sugieren que la terapia láser de baja potencia favorece la respuesta del hueso alveolar dañado.

PALABRAS CLAVE: Laserterapia de baja potencia; Láser bioestimulación; Hueso alveolar.

\section{INTRODUCCIÓN}

La terapia con Láser de Baja Potencia (LBP), del inglés Low Level Laser Therapy (LLLT), ha demostrado tener un efecto analgésico, antiinflamatorio y bioestimulante a través de un incremento del trofismo celular y de la microcirculación local, acelerando la velocidad de cicatrización de heridas, así como la reducción de edema e inflamación post-operatoria (Tunér \& Hode, 1998). Suazo et al. (2007) demostraron que la aplicación de láser de baja potencia producía cambios microvasculares en el tejido conectivo, observándose un aumento de la densidad microvascular.

En odontoestomatología, las principales aplicaciones del láser de baja potencia son en hipersensibilidad dentinaria, lesiones aftosas y herpéticas, neuralgia del trigémino, disfunción de ATM, parálisis facial, lesiones periapicales, bioestimulación ósea, etc. (Oltra-Arimon et al., 2004).

Se ha postulado que el LBP puede acelerar los procesos de reparación ósea, debido al efecto antiinflamatorio que se produce al normalizar la microcirculación y activar la fagocitosis, a la vez que estimula la proliferación celular aumentando la capacidad reparativa del hueso. Estudios han demostrado que el LBP puede promover la curación y mineralización del hueso, siendo clínicamente beneficioso en la promoción de la formación de hueso en defectos esqueléticos (Lirani-Galvão et al., 2006; Merli et al., 2005).

Diversos estudios han demostrado un aumento en la velocidad de reparación del tejido óseo; primero en animales de experimentación y posteriormente con pacientes que presentaban fracturas (Loumitzky, 1985; Katz, 1985).

Para Yamada (1991) el LBP promueve la síntesis de DNA y la proliferación de los osteoblastos en cultivo, los cuales se mantienen normales y en fase activa con dosis de hasta $10 \mathrm{~J} / \mathrm{cm} 2$, pero al aumentar a $30 \mathrm{~J} / \mathrm{cm}^{2}$ presentan signos de degradación e inclusive de destrucción total.

Silva Júnior et al. (2002) demostraron en ratones que la aplicación diaria de LBP por más de siete días acelera la

\footnotetext{
Unidad de Anatomía Normal, Facultad de Ciencias de la Salud, Universidad de Talca, Chile.

** Departamento de Estomatología, Facultad de Ciencias de la Salud, Universidad de Talca, Chile.
} 
neoformación trabecular y a los cinco días se observa una mayor aposición de minerales. Barushka et al. (1995) y Dickson et al. (1994) indicaron que el LBP aumenta la actividad y el número de osteoblastos y osteoclastos, así como un incremento de la actividad de fosfatasa alcalina, mientras Lirani \& Lazaretti-Castro (2005) indicaron que el LBP aumenta la expresión de la osteocalcina, proteína que participa en la migración, proliferación y diferenciación de células osteoblásticas.

Similares resultados fueron reportados por Stein et al. (2005) y Martinasso et al. (2007), quienes indicaron que en el hueso sometido a LBP existe un aumento en la expresión de marcadores osteogénicos como la fosfatasa alcalina, la osteopontina y la sialoproteina ósea. Análisis histométricos en ratones revelaron una concentración de hueso neoformado mas rápida en ratas sometidas a LBP (Pourzarandian et al., 2004; Merli et al.; Lirani \& Lazaretti-Castro), con resultados significativos a partir del segundo día de LBP (Osawa et al., 1998). El propósito de este estudio fue analizar el efecto sobre el número de osteonas del LBP, aplicado sobre el hueso alveolar dañado.

\section{MATERIAL Y MÉTODO}

Se utilizaron 13 ratas Sprague Dawley, adultas hembras, de 250 a 300 grs. Las cuales fueron adquiridas en el bioterio ANEXPA de la ciudad de Talca. Durante todo el periodo experimental se alojaron en jaulas, mantenidos con ciclos de 12-12 hrs. Luz-oscuridad, con calefacción y con una dieta estándar de alimentos sólidos (pellet) y agua potable ad libitum.

Las ratas fueron sometidas a anestesia inhalatoria con éter etílico en una cámara de anestesia. Se consideró que la rata estaba correctamente anestesiada cuando se lograba una respiración toracoabdominal y ausencia de reflejo de los bigotes. Una vez inducida la anestesia, y en condiciones de asepsia, se infiltró una solución de lidocaína al 3\% y luego se realizó una incisión en la mucosa vestibular frente al incisivo central inferior derecho, desprendiéndose un colgajo mucoperióstico que permitió realizar una osteotomía estandarizada de $0.8 \mathrm{~mm} 3$ de hueso para lo cual se utilizó una fresa esférica de Carbide de $0.8 \mathrm{~mm}$ de diámetro.

Una muestra aleatoria de 7 ratas fue sometida a un protocolo de aplicación de láser frente al alveolo en cicatrización en dosis de $6 \mathrm{~J} / \mathrm{cm} 2$. Procedimiento que se repitió tres veces por semana durante cuatro semanas. El resto de las ratas se mantuvo como control.
Terminado el protocolo de irradiación, se sacrificó a las ratas mediante una sobre dosis inhalatoria de éter etílico. Luego las cabezas fuero seccionadas y las mandíbulas diseccionadas para ser fijadas con formalina al 10\%. Se obtuvieron bloques de $10 \mathrm{~mm}^{3}$ de la zona lesionada, los que fueron procesados para hematoxilina-eosina, obteniéndose cortes semiseriados de $5 \mu \mathrm{m}$, los cuales fueron analizados con microscopio óptico con aumento de 40x, contabilizándose el número de osteonas presentes en el campo. La muestra fue analizada por dos observadores, y se estableció la concordancia interobservadores.

La información fue recopilada en las fichas de recolección de datos y mediante el programa estadístico STATA 9.0 para Windows se calcularon los estadísticos descriptivos. Para determinar la significancia estadística de las diferencias se utilizó la prueba t para muestras independientes con un nivel de confianza del $99 \%$. Sobre la base de una media de las osteonas totales contabilizadas con un intervalo de confianza del $95 \%$ se calculó la fuerza de la asociación estadística mediante los odds ratio.

\section{RESULTADOS}

Se analizaron un total de 83 cortes histológicos, 41 para el grupo Control y 42 para el grupo Experimental. Para contar la cantidad de osteonas secundarias en cada muestra se realizó el conteo por campo visual. La media del osteonas encontradas en el grupo control fue de 62,29 (DS 22,802), mientras que la media en el grupo de ratas sometidas a LLLT fue de 104,29 (DS 48,025). Estos resultados se grafican en la Fig. 1.

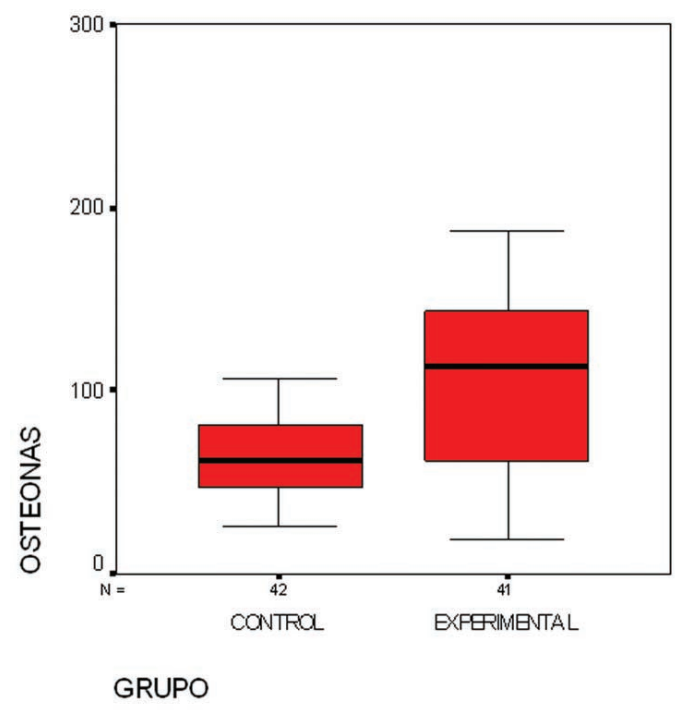

Fig. 1. Diagrama de cajas que grafica el número de osteonas en el hueso alveolar de ratas sometidas aláser de baja potencia. 
La diferencia de medias en el número de osteonas encontradas en los grupos control y experimental resultó estadísticamente significativas $(\mathrm{p}<0,01)$. Para la variable osteonas totales contabilizadas la media fue de 83,036 , con un intervalo de confianza $[73,69-92,38]$ al $95 \%$. Se encontró una alta asociación estadística de la diferencia, con un odds ratio de 5,6, con un intervalo de confianza [1,96-16,5] al $95 \%$, por lo que por cada individuo sin terapia que tiene un número de osteonas superior a la media existen 5,6 individuos que tienen un número de osteonas superior a la media con uso de láser.

La concordancia interobservadores fue de un k: 0,95 para el grupo Experimental y un k:0,96 para el grupo Control.

\section{DISCUSIÓN}

El efecto bioestimulador del LBP sobre las células es conocido y ampliamente documentado desde el inicio de su uso en las ciencias medicas (Martelli et al., 2002). El láser de baja potencia además de carecer del efecto térmico que posee el láser duro o de alta potencia, posee un efecto analgésico, antiinflamatorio y bioestimulante generando un aumento del trofismo celular y de la microcirculación local, acelerando la cicatrización de heridas, así como la reducción de edema e inflamación post-operatoria tanto en tejidos duros como blandos.

En el presente estudio se analizaron los cambios morfológicos del hueso alveolar asociados a la aplicación de terapia con láser de baja potencia durante la cicatrización. Mediante el análisis histológico de las muestras obtenidas se observó que a dos meses de realizada la lesión en el hueso y a la posterior aplicación de LBP durante el primer mes, existieron cambios morfológicos de importancia, como es el aumento en el número promedio de osteonas en el grupo experimental o irradiado (Media 104,29) en comparación con el grupo no irradiado (Media 62,29), lo cual resultó estadísticamente significativo $(\mathrm{p}<0,001)$ en concordancia con lo encontrado por Silva Júnior et al., quienes observaron que la aplicación por más de 7 días de LBP aumenta la neoformación trabecular y la aposición de minerales.

Los resultados de este estudio confirman lo observado por Oltra-Arimon et al., quienes concluyeron que el LBP produce la bioestimulación ósea promoviendo la reparación y remodelado óseo. Similares resultados obtuvo Merli et al., Lirani-Galvão et al. y Suazo et al., quienes encontraron que la estimulación con LBP acelera el proceso de reparación ósea, normaliza la microcirculación activa de la fagocitosis, aumentando la proliferación celular, la capacidad reparativa del hueso y la mineralización del hueso neoformado.

$\mathrm{Al}$ analizar las muestras histológicas se comprueba que la estimulación con láser de baja potencia sobre el hueso alveolar en cicatrización genera cambios morfológicos, como el aumento del número de osteonas y vasos sanguíneos, como ha sido reportado por diversos autores (Merli et al.; Lirani \& Lazaretti-Castro), estos antecedentes aportan evidencias de los efectos benéficos que la utilización del LBP puede tener en el manejo de defectos o lesiones en el hueso alveolar.

LANDAETA, B. M. J.; SUAZO, G. I. C.; CANTIN, L. M.; ROA, H. I. J.; ZAVANDO, M. D. A. The low level laser therapy effect on alveolar bone damaged. Int. J. Morphol., 26(3):639-642, 2008.

SUMMARY: The Low Level Laser Therapy (LLLT) has demostrated to have analgesic, antiinflamatory, bioestimulant and promoters from the tissues responses properties to the damage. The purpose of this study was determinate the Low Level Laser Therapy effect in the damaged alveolar bone. Thirteen Sprage Dawley rats were used. Total number of animals alveolar bones a standarized lesion was made, later an aleatory sample of seven rats was subjected to the irradiation protocol $6 \mathrm{~J} / \mathrm{cm}^{2}$, three times per week during four weeks. The obtained samples of the injured area, of exposed and not exposed rats to the laser therapy were processed for hematoxilin \& eosin, being the osteon number count by optic microscope whith increase of 40x. The result show an increase in the osteon number in the irradiated group, this differentiated was statistically significant $(\mathrm{p}<0.01)$, whit a high strength of statistical association $(\mathrm{OR}=5.6)$. These result suggest that the therapy laser of low power favors the answer of the damaged alveolar bone.

KEY WORDS: Low level laser therapy; Laser biostimulation; Alveolar bone.

\section{REFERENCIAS BIBLIOGRÁFICAS}

Barushka, O.; Yaakobi, T. \& Oron, U. Effect of low-energy laser $(\mathrm{He}-\mathrm{Ne})$ irradiation on the process of bone repair in the rat tibia. Bone, 16(1):47-55, 1995.
Dickson, G. R.; Clingen, H.; Jordan, G. R. \& Linton, T. The effect of low level laser therapy on alkaline phosphatase expresion during fracture repair. Laser The., 6:16-7, 1994. 
Katz, A. G. Tratamiento con láser en fracturas del maxilar inferior. Stom., 64(2):57-9, 1985.

Lirani-Galvão, A. P.; Jorgetti, V. \& da Silva, O. L. Comparative study of how low-level laser therapy and low-intensity pulsed ultrasound affect bone repair in rats. Photomed. Laser Surg., 24(6):735-40, 2006.

Lirani, A. P. \& Lazaretti-Castro, M. Evidences of physical agents action on bone metabolism and their potential clinical use. Arq. Bras. Endocrinol. Metabol., 49(6):8916, 2005.

Loumitzky, N. Características clínico-radiológicas de la cicatrización en fracturas mandibulares después del empleo del láser helio-néon. Stom., 64(3):38-9, 1985.

Martelli, F. S.; Sáez de la Fuente, I. \& Nisio, A. Aplicaciones clínicas del láser en implantología. Rev. Esp. odontoestomatológica de implantes, 2(10):60-5, 2002.

Martinasso, G.; Mozzati, M.; Pol, R.; Canuto, R. A. \& Muzio, G. Effect of superpulsed laser irradiation on bone formation in a human osteoblast-like cell line. Minerva Stomatol., 56(1-2):27-30, 2007.

Merli, L. A.; Santos, M. T.; Genovese, W. J. \& Faloppa, F. Effect of low-intensity laser irradiation on the process of bone repair. Photomed. Laser Surg., 23(2):212-5, 2005.

Oltra-Arimon, D.; España-Tost, A.; Berini-Aytés, L. \& GayEscoda, C. Aplicaciones del láser de baja pótencia en Odontología. RCOE, 9(5):517-24, 2004.

Ozawa, Y.; Shimizu, N.; Kariya, G. \& Abiko, Y. Low-energy laser irradiation stimulates bone nodule formation at early stages of cell culture in rat calvarial cells. Bone, 22(4):347-54, 1998.

Pourzarandian, A.; Watanabe, H.; Aoki, A.; Ichinose, S.; Sasaki, K. M.; Nitta, H. \& Ishikawa, I. Histological and TEM examination of early stages of bone healing after Er:YAG laser irradiation. Photomed. Laser Surg., 22(4):342-50, 2004.

Silva Júnior, A. N.; Pinheiro, A. L.; Oliveira, M. G.; Weismann, R.; Ramalho, L. M. \& Nicolau, R. A. Computerized morphometric assessment of the effect of low-level laser therapy on bone repair: an experimental animal study. J. Clin. Laser Med. Surg., 20(2):83-7, 2002.

Stein, A.; Benayahu, D.; Maltz, L. \& Oron, U. Low-level laser irradiation promotes proliferation and differentiation of human osteoblasts in vitro. Photomed. Laser Surg., 23(2):161-6, 2005.

Suazo, G. I. C.; Lara, S. M. C.; Cantín, L. M. G. \& Zavando, M. D. A. Efecto de la aplicación de láser de baja potencia sobre la mucosa oral lesionada. Int. J. Morphol; 25(3):523-8, 2007.

Tunér, J. \& Hode, L. It's all in the parameters: a critical analysis of some well-known negative studies on lowlevel laser therapy. J. Clin. Laser. Med. Surg., 16(5):2458, 1998.

Yamada, K. Biological effects of low power laser irradiation on clonal osteoblastic cells (MC3T3-E1). Nippon Seikeigeka Gakkai Zasshi. 65(9):787-99, 1991.

Dirección para correspondencia: Iván Claudio Suazo Galdames Unidad de Anatomía Normal Universidad de Talca Avenida Lircay s/n oficina N¹04 Talca -CHILE

Fono 56-71-201682

Email: isuazo@utalca.cl

Recibido : 24-10-2007

Aceptado: 16-07-2008 